\title{
Detección de anticuerpos anti-Mycobacterium avium subsp. paratuberculosis (MAP) en venados silvestres en Colombia
}

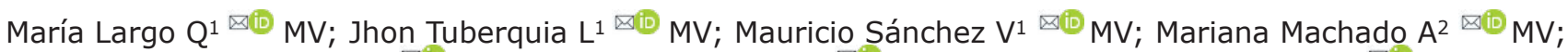

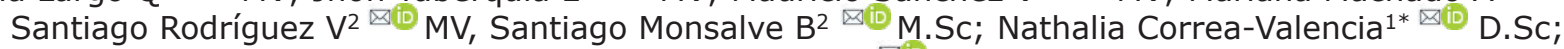
Jorge A. Fernández-Silva, ${ }^{\otimes(\mathbb{D}}$ Dr.Med.Vet.

${ }^{1}$ Universidad de Antioquia, Facultad de Ciencias Agrarias, Escuela de Medicina Veterinaria, Grupo Centauro, Medellín, Colombia.

${ }^{2}$ Corporación Universitaria Lasallista, Grupo GIVET, Caldas, Colombia.

*Correspondencia: mariadelp.correa@udea.edu.co

Recibido: Octubre 2019; Aceptado: Febrero 2020; Publicado: Julio 2020.

\section{RESUMEN}

Objetivo. Determinar la presencia de anticuerpos anti-Mycobacterium avium subsp. paratuberculosis (MAP) en venados de cola blanca (Odocoileus virginianus) y venados de páramo (Mazama rufina), capturados en las regiones de la Orinoquía y el Caribe en Colombia. Materiales y métodos. Un total de 44 muestras de suero sanguíneo de venados de las especies $M$. rufina y $O$. virginianus fueron colectadas en condiciones de campo entre 2014 y 2016. Se utilizó un kit comercial de ELISA para la detección de anticuerpos anti-MAP. Un animal se consideró positivo a ELISA cuando la relación muestrapositivo (S/P\%) fue $\geq 0.4$, según lo recomendado por el fabricante. Resultados. El 50\% (22/44) de los animales muestreados resultaron positivos, lo que corresponde a 10 hembras y 12 machos. Un $81.8 \%(18 / 22)$ y un $77.3 \%(17 / 22)$ de estos animales seropositivos fueron capturados en la región de la Orinoquía y fueron reportados como adultos, respectivamente. Conclusiones. No se sabe cómo o cuándo se introdujo MAP en la población de ciervos colombianos en las regiones de estudio. La hipótesis más plausible para explicar la presencia de anticuerpos anti-MAP en estas poblaciones silvestres es la transmisión por contacto con el ganado bovino infectado, ya que en ambas regiones estas especies comparten pasturas. Este es el primer estudio en explorar la infección por MAP en animales silvestres en Colombia. Estos hallazgos respaldan la necesidad de realizar más estudios utilizando técnicas de diagnóstico directo, y aproximaciones investigativas que permitan la definición de vínculos en la dinámica de la infección entre mamíferos silvestres y domésticos en Colombia.

Palabras clave: Cérvidos; ELISA; enfermedad de Johne; micobacteria; rumiantes silvestres (Fuentes: DeCS, CAB).

\section{ABSTRACT}

Objective. To determine the presence of anti-Mycobacterium avium subsp. paratuberculosis (MAP) antibodies in white-tailed deer (Odocoileus virginianus) and red brocket deer (Mazama rufina), captured in the Orinoquía and Caribbean regions in Colombia. Materials and methods. A total of 44 blood serum samples from deer of species $M$. rufina and $O$. virginianus were collected under

Como citar (Vancouver):

Largo QM, Tuberquia LJ, Sánchez VM, Machado AM, Rodríguez VS, Monsalve S, Correa-Valencia N, Fernández-Silva J. Detección de anticuerpos anti-Mycobacterium avium subsp. paratuberculosis (MAP) en venados silvestres en Colombia. Rev MVZ Cordoba. 2020; 25(2):e1809. https://doi.org/10.21897/rmvz.1809 
field conditions between 2014 and 2016. An ELISA commercial kit was used to detect anti-MAP antibodies. An animal was considered ELISA-positive at a sample-to-positive ratio (S/P\%) of $\geq 0.4$, as recommended by the manufacturer. Results. The $50 \%(22 / 44)$ of the animals were positive, corresponding to 10 females and 12 males. An 81.8\% (18/22) and 77.3\% (17/22) of these seropositive animals were captured in the Orinoquía region and were reported as adults, respectively. Conclusions. It is not known how or when MAP was introduced in the Colombian deer population in the study regions. The most plausible hypothesis to explain the presence of antibodies against MAP in these wild populations is transmission by contact with infected bovine cattle since, in both regions, these species share pastures. This is the first study to explore MAP infection in wild animals in Colombia. These findings support the need for further studies using different direct diagnostic techniques and research approaches that allow the definition of links in the infection dynamics between wild and domestic mammals in Colombia.

Keywords: Cervids; ELISA; Johne's disease; micobacteria; wild ruminants (Fuentes: DeCS, CAB).

\section{INTRODUCCIÓN}

Mycobacterium avium subsp. paratuberculosis (MAP) forma parte del complejo Mycobacterium avium (MAC) y es el agente etiológico de una gastroenteritis crónicas en rumiantes conocida como paratuberculosis (PTB) o enfermedad de Johne (JD). La PTB causa lesiones inflamatorias en las paredes de yeyuno e íleon, afectando la absorción de nutrientes y proteínas, lo que conduce a la pérdida de masa muscular y a una menor productividad. Los bovinos afectados presentan diarrea, edema submandibular, pérdida de peso (a pesar del apetito normal), letargo y muerte. En general, los signos clínicos de la PTB en cérvidos son similares a los observados en el ganado bovino, a saber, diarrea crónica, pérdida de peso y disminución de la condición corporal $(1,2)$. La PTB conduce a pérdidas económicas para los sistemas ganaderos debido al sacrificio prematuro, costos de reemplazo, reducción de la producción de leche y ganancia de peso, trastornos de fertilidad y una mayor susceptibilidad a otras enfermedades (3).

La posible asociación de MAP con la enfermedad de Crohn (EC) y otras enfermedades humanas, refleja el potencial zoonótico del agente, reconocido como asociado a MAP (4).

Adicionalmente, la PTB afecta a especies domésticas y de fauna silvestre en todo el mundo (5). Se ha confirmado la presencia de MAP en 178 especies de fauna silvestre cautivas y en libertad (6). En América Latina existen reportes al respecto. Un estudio en heces de guanacos salvajes (Lama guanicoe) reportó el aislamiento de MAP, utilizando técnicas microbiológicas y moleculares (7). En 2013, el primer caso de infección por Mycobacterium intracellulare, miembro del complejo MAC, se informó en un capibara (Hydrochoerus hydrochaeris) con lesiones granulomatosas típicas de micobacteriosis en Argentina, lo que sugiere la susceptibilidad a la infección por otras bacterias del complejo MAC, como MAP (8). En Perú, se colectaron muestras de vicuñas cautivas y en libertad (Vicugna vicugna mensalis) y otros camélidos sudamericanos (Vicugna pacos y Lama glama), reportando resultados serológicos negativos a MAP (9). En un estudio más reciente, se reportó la presencia de MAP en heces de alpacas chilenas ( $V$. pacos), usando cultivo, reacción en cadena de la polimerasa (PCR) y técnicas de genotipado (10). En Colombia, la PTB se reportó por primera vez en el ganado bovino en 1924. Desde entonces la enfermedad se ha explorado principalmente en bovinos y ovinos, pero no hay información epidemiológica disponible sobre MAP en animales silvestres hasta el momento (11).

El venado de páramo (Mazama rufina) es un pequeño ciervo, nativo de los Andes colombianos, Ecuador y del norte de Perú, presente en ambientes de bosque y páramo. El venado de cola blanca (Odocoileus virginianus) tiene una distribución geográfica más amplia (de Canadá a Perú) y ocho subespecies son reconocidas en América del Sur. Las especies $M$. rufina y $O$. virginianus proporcionan una fuente alternativa de proteínas en los países en desarrollo y son especies altamente cazadas, a pesar de ser una práctica prohibida en Colombia (12).

El objetivo del presente estudio fue determinar la presencia de anticuerpos anti-MAP en venados 
de cola blanca ( $O$. virginianus) y venados de páramo ( $M$. rufina), capturados en condiciones de campo en las regiones de la Orinoquía y Caribe en Colombia.

\section{MATERIALES Y MÉTODOS}

Aspectos éticos. El comité de ética para la experimentación animal (CEEA) de la Universidad de Antioquia, Colombia (Acta \# 111, junio de 2017) aprobó este estudio. La Autoridad Nacional de Licencias Ambientales (ANLA), Colombia (Auto \# 00852, marzo de 2017) aprobó la recolección de muestras.

Animales y muestras. Se colectó sangre en un muestreo a conveniencia de 44 individuos silvestres de venado de páramo ( $M$. rufina) y venado de cola blanca (O. virginianus), capturados en condiciones de campo en las regiones de la Orinoquía y Caribe colombianas, entre 2014 y 2016. Un total de 22 animales fueron capturados y muestreados en cada una de las regiones, respectivamente. Los 22 animales correspondientes a la región Caribe fueron capturados y muestreados en los departamentos de Córdoba $(n=12)$, Sucre $(n=6)$, Bolívar $(n=2)$ y Magdalena $(n=2)$. A su vez, los venados del departamento de Córdoba fueron capturados y muestreados en los municipios de Montería y San Pelayo, mientras que los venados los departamentos de Sucre, Bolívar y Magdalena fueron capturados y muestreados en los municipios de Sincelejo, Magangué y Santa Ana, respectivamente. Por su parte, los 22 animales correspondientes a la región de la Orinoquía fueron capturados y muestreados en el municipio de Saravena, departamento de Arauca.

Los sueros se obtuvieron usando una centrífuga manual, a $3.000 \mathrm{rpm}$, y fueron transportados en nitrógeno líquido al laboratorio donde se almacenaron a $-80^{\circ} \mathrm{C}$ hasta su análisis.

Ensayo por inmunoabsorción ligado a enzimas (ELISA). Las muestras de suero se analizaron utilizando un kit comercial de ELISA indirecto pre-absorbido y de acuerdo con las instrucciones del fabricante (Cattletype ${ }^{\circledR}$ MAP, Qiagen Leipzig GmbH, Leipzig, Alemania). Los resultados se consideraron válidos si el valor medio (VM) del valor OD medido para el control positivo (PC) era $\geq 0.7$ y si el valor medido del valor OD para el control negativo (NC) era $\leq 0.2$. Un animal se consideró positivo para ELISA cuando la relación muestra/positivo (S/P\%) era $\geq 0.4$, según lo recomendado por el fabricante.
Análisis de datos. Toda la información generada durante el estudio se ingresó en hojas de cálculo de Excel (Microsoft Corp., Redmond, WA, EE. UU.) y luego se exportó a Stata 14.0 (StataCorp, 2017, Texas, EE. UU.) para su análisis estadístico. Se calcularon las estadísticas descriptivas para todas las variables (i.e. ubicación de captura, sexo, grupo etario), y se exploró la asociación entre dichas variables y el resultado serológico $(p<0.05)$, mediante la prueba de chi-cuadrado de Pearson.

\section{RESULTADOS}

El 54.5\% (24/44) de los venados muestreados eran hembras, mientras que el $45.5 \%$ (20/44) eran machos. El cincuenta por ciento (22/44) de Ios animales resultaron positivos a la presencia de anticuerpos anti-MAP. El 100\% $(n=22)$ de los animales positivos pertenecen a la especie O. virginianus, 10 de los cuales eran hembras y 12 , machos. El $18.2 \%(4 / 22)$ de los animales detectados como seropositivos fueron capturados en la región Caribe, mientras que el $81.8 \%$ $(18 / 22)$, en la región de la Orinoquía. Al análisis estadístico no se encontraron asociaciones entre las variables de estudio y el resultado obtenido mediante ELISA. Las características de la población muestreada, en cuanto al resultado serológico obtenido, se presentan en la tabla 1.

Tabla 1. Resultados de la prueba de ELISA para la detección de anticuerpos anti-Mycobacterium avium subsp. paratuberculosis en venados silvestres (Mazama rufina y Odocoileus virginianus) de Colombia.

\begin{tabular}{cccccc}
\hline \multirow{2}{*}{ Región } & Departamento & Municipio & \multicolumn{4}{c}{ Resultado del ELISA } \\
\cline { 3 - 6 } & \multirow{4}{*}{ Córdoba } & Montería & 4 & 4 & 8 \\
& & San Pelayo & 0 & 4 & 4 \\
\multirow{2}{*}{ Caribe } & Sucre & Sincelejo & 0 & 6 & 6 \\
& Bolívar & Magangué & 0 & 2 & 2 \\
\multirow{2}{*}{ Orinoquía } & Magdalena & Santa Ana & 0 & 2 & 2 \\
& Arauca & Saravena & 18 & 4 & 22 \\
\hline \multirow{2}{*}{} & Total & & 22 & 22 & 44 \\
\hline
\end{tabular}

\section{DISCUSIÓN}

El presente estudio tuvo como objetivo determinar la presencia de anticuerpos anti-MAP detectados por ELISA en venados silvestres capturados en dos regiones naturales de Colombia. Según el 
conocimiento de los autores, este es el primer estudio que explora la infección por MAP en animales silvestres en el país. La seropositividad encontrada (50\%) pudo verse influenciada por el método de muestreo que se realizó, el cual fue a conveniencia y no permite obtener una muestra representativa de la población. Es también importante tener en cuenta que los rumiantes pueden desarrollar una enfermedad sin respuesta de anticuerpos o una respuesta inconsistente contra MAP (13). Dichas particularidades podrían haber afectado la frecuencia de infección por MAP reportada aquí, debido a resultados falsos negativos.

En cualquier caso, las altas tasas de seropositividad en animales para el consumo humano es una preocupación en salud pública, ya que son mayores las probabilidades de que los animales infectados puedan transmitir la bacteria a la cadena alimenticia humana a través de la carne contaminada con MAP (14). Más aún cuando en Colombia la carne producto de la caza no está supervisada por la autoridad sanitaria, el instituto nacional de la vigilancia de medicamentos y alimentos (INVIMA).

La hipótesis más plausible para explicar la presencia de anticuerpos anti-MAP en venados en Colombia, es la ingestión residual de la bacteria a partir de un ambiente previamente contaminado con heces de rumiantes domésticos o silvestres infectados, e incluso animales no rumiantes que comparten dicho hábitat, como se ha reportado en otro estudios $(5,6,15)$. En la región estudiada es muy común que los rumiantes domésticos compartan pastos $y$ recursos hídricos con especies de vida silvestre. Bajo ciertas condiciones, el medio ambiente juega un papel importante en la transmisión de MAP dentro de las especies, que puede a su vez contribuir a la supervivencia de la bacteria hasta por un año en el suelo e incluso más tiempo en agua (16). Por otro lado, la transmisión de bacterias del género Mycobacterium entre el ganado y las especies silvestres, incluidos los venados silvestres, ha sido sugerida por varios autores y puede explicarse por el hecho de que comparten fuentes de alimento y agua $(15,17)$. Sin embargo, las dinámicas asociadas con la población bovina de la zona no pueden ser exploradas, ya que no existen reportes de MAP/ PTB en la zona de interés al día de hoy (11).
De acuerdo con el instituto colombiano agropecuario (ICA) -ente público encargado de la prevención, vigilancia y control de los riesgos sanitarios, biológicos y químicos para las especies animales y vegetales, las regiones de estudio reúnen el 30 y el $21 \%$ de la población bovina en Colombia, respectivamente. Esta distribución muestra la importante participación de estas dos regiones en la producción nacional bovina y el posible impacto que pueden tener en los ecosistemas donde se desarrollan. El sistema de ganadería extensiva en estas dos regiones naturales de Colombia se caracteriza por tener una baja densidad de ganado (aproximadamente 1.5-1.8 animales/Ha). Además, es una práctica común que el ganado pastoree libremente en llanuras naturales sin cercas, por lo que es probable el contacto directo con los venados silvestres o con sus heces.

Estos hallazgos respaldan la necesidad de realizar más estudios utilizando técnicas de diagnóstico directo (bacteriológicas y moleculares), y aproximaciones investigativas que permitan la definición de vínculos en la dinámica de la infección entre mamíferos silvestres y domésticos en Colombia, considerando factores de riesgo específicos a nivel de las poblaciones involucradas en los sistemas extensivos de ganado colombiano. También es necesario aclarar el papel de los venados en la epidemiología de MAP, para esto es necesario el uso de herramientas como el genotipado que permiten rastrear las fuentes y los patrones de transmisión. El nivel de diversidad genética obtenido mediante la combinación de técnicas de genotipado (i.e. MIRU-VNTR, MLSSR, SNPs) es una vía prometedora para las investigaciones de epidemiología molecular de este patógeno (18).

\section{Conflicto de intereses}

Los autores no tienen conocimiento de ninguna relación financiera o personal con otras personas u organizaciones que puedan influir de manera inapropiada en el trabajo que se informa en este documento.

\section{Agradecimientos}

Los autores agradecen al comité para el desarrollo de la investigación (CODI), Universidad de Antioquia (Medellín, Colombia) por el apoyo financiero al proyecto de investigación. 


\section{REFERENCIAS}

1. Palmer $M$ V, Kanipe $C$, Cox R, RobbeAusterman S, Thacker TC. Characteristics of subclinical Mycobacterium avium ssp. paratuberculosis infection in a captive white-tailed deer herd. J Vet Diagnostic Investig. 2019; 31(6):844-51. https://doi. org/10.1177\%2F1040638719873028

2. Sleeman JM, Manning EJB, Rohm JH, Sims JP, Sanchez S, Gerhold RW, et al. Johne's disease in a free-ranging whitetailed deer from Virginia and subsequent surveillance for Mycobacterium avium subspecies paratuberculosis. J Wildl Dis. 2009; 45(1):201-206. https://doi. org/10.7589/0090-3558-45.1.201

3. Garcia AB, Shalloo L. Invited review: The economic impact and control of paratuberculosis in cattle. J Dairy Sci. 2015; 98(8):5019-5039. https://doi.org/10.3168/ jds.2014-9241

4. Kuenstner JT, Naser S, Chamberlin W, Borody T, Graham DY, McNees A, et al. The Consensus from the Mycobacterium avium ssp. paratuberculosis (MAP) Conference 2017. Front Public Heal. 2017; 5(208):1-5. https://doi.org/10.3389/fpubh.2017.00208

5. Stevenson K, Àlvarez J, Bakker D, Biet F, Juan L, Denham S, et al. Occurrence of Mycobacterium avium subspecies paratuberculosis across host species and European countries with evidence for transmission between wildlife and domestic ruminants. BMC Microbiol. 2009; 9(212):113. https://doi.org/10.1186/1471-2180-9$\underline{212}$

6. Carta T, Álvarez J, Pérez de la Lastra JM, Gortázar C. Wildlife and paratuberculosis: A review. Res Vet Sci. 2013; 94(2):191-197. https://doi.org/10.1016/j.rvsc.2012.11.002

7. Salgado M, Herthnek D, Bolske G, Leiva S, Kruze J. First isolation of Mycobacterium avium subsp. paratuberculosis from wild guanacos (Lama guanicoe) on Tierra del Fuego Island. J Wildl Dis. 2009; 45(2):295301. https://doi.org/10.7589/0090-3558$\underline{45.2 .295}$
8. Pezzone $\mathrm{N}$, Eberhardt AT, Fernández $\mathrm{A}$, Garbaccio S, Zumárraga M, Gioffré $A$, et al. Mycobacterium intracellulare infection in a capybara (Hydrochoerus hydrochaeris). J Zoo Wildl Med. 2013; 44(4):1098-1101. https://doi.org/10.1638/2013-0017R1.1

9. Risco-Castillo V, Wheeler JC, Rosadio R, Garcia-Pena FJ, Arnaiz-Seco I, Hoces D, et al. Health impact evaluation of alternative management systems in vicuna (Vicugna vicugna mensalis) populations in Peru. Trop Anim Health Prod. 2014; 46(4):641-646. https://doi.org/10.1007/s11250-014-0543$\underline{3}$

10. Salgado M, Sevilla I, Rios C, Crossley J, Tejeda C, Manning E. Presence of Mycobacterium avium subsp. paratuberculosis in alpacas (Lama pacos) inhabiting the Chilean Altiplano. J Zoo Wildl Med. 2016; 47(1):1216. https://doi.org/10.1638/2012-0255.1

11. Correa-Valencia N, García-Tamayo YM, Fernández-Silva JA. Mycobacterium avium subsp. paratuberculosis in Colombia (19242016): A review. Rev Colomb Cienc Pec. 2018; 31:165-179. https://doi. org/10.17533/udea.rccp.v31n3a01

12. Weber M, Gonzalez S. Latin American deer diversity and conservation: A review of status and distribution. Écoscience. 2003; $10(4): 443-454$. https://doi.org/10.1080/1 1956860.2003.11682792

13. Munster $P$, Fechner $K$, Volkel $I$, von Buchholz A, Czerny C-P. Distribution of Mycobacterium avium ssp. paratuberculosis in a German zoological garden determined by IS900 semi-nested and quantitative real-time PCR. Vet Microbiol. 2013; 163(12):116-123. https://doi.org/10.1016/j. vetmic. 2012.12.009

14. Gill CO, Saucier L, Meadus WJ. Mycobacterium avium subsp. paratuberculosis in dairy products, meat, and drinking water. J Food Prot. $2011 ; 74(3): 480-99$. https://doi. org/10.4315/0362-028X.JFP-10-301 
15. Galiero A, Leo S, Garbarino C, Arrigoni N, Russo S, Giacomelli S, et al. Mycobacterium avium subsp. paratuberculosis isolated from wild red deer (Cervus elaphus) in Northern Italy. Vet Microbiol. 2018; 217:167-172. https://doi.org/10.1016/j. vetmic.2018.03.015

16. Whittington RJ, Marshall DJ, Nicholls PJ, Marsh IB, Reddacliff LA. Survival and dormancy of Mycobacterium avium subsp. paratuberculosis in the environment. Appl Environ Microbiol. 2004; 70(5):2989-3004. https://doi.org/10.1128/aem.70.5.29893004.2004
17. Albertti LAG, Souza-Filho AF, Fonseca-Júnior $A A$, Freitas $M E$, de Oliveira-Pellegrin $A$, Zimmermann NP, et al. Mycobacteria species in wild mammals of the Pantanal of central South America. Eur J Wildl Res. 2015; 61(1):163-166. https://doi.org/10.1007/ s10344-014-0866-4

18. Ahlstrom C, Barkema HW, Stevenson $\mathrm{K}$, Zadoks RN, Biek R, Kao R, et al. Genome-wide diversity and phylogeography of Mycobacterium avium subsp. paratuberculosis in Canadian dairy cattle. PLoS One. 2016; 11(2):e0149017. https:// doi.org/10.1371/journal.pone.0149017 